\title{
Evaluación del Sistema de Tracción en un Vehículo Eléctrico Biplaza de Estructura Tubular
}

\author{
Juan Rocha-Hoyos ${ }^{(1,3)}$, Luis E. Tipanluisa ${ }^{(2)}$, Salvatore W. Reina ${ }^{(2)}$ y César R. Ayabaca ${ }^{(2)}$ \\ (1) Facultad de Arquitectura e Ingenierías, Universidad Internacional SEK, Casilla 17-03-02, \\ Quito - Ecuador (e-mail: carlos.rocha@uisek.edu.ec) \\ (2) Dpto. de Ingeniería Mecánica, Facultad de Ingeniería Mecánica, Escuela Politécnica Nacional, \\ Ladrón de Guevara, E11-253, P.O. Box 17-01-2759. Quito - Ecuador (e-mail: luis.tipanluisa@epn.edu.ec) \\ (3) Departamento de Energía y Mecánica, Universidad de las Fuerzas Armadas ESPE, Sangolquí - \\ Ecuador
}

Recibido May. 30, 2016; Aceptado Jul. 25, 2016; Versión final Sep. 28, 2016, Publicado Abr. 2017

\begin{abstract}
Resumen
En este artículo se presenta una alternativa a la contaminación de los vehículos de gasolina tipo go-kart $7.5 \mathrm{~kW}$ de la ciudad de Baños en Ecuador, con el fin de reducir las emisiones contaminantes. La propuesta es un prototipo de vehículo eléctrico tubular de dos asientos que responden a requisitos de diseño tales como: capacidad máxima de $420 \mathrm{~kg}$, con una media de velocidad $50 \mathrm{~km} / \mathrm{h}$, subir pendientes de $14 \%$, y mantener el confort de los ocupantes. La topografía de la ciudad ha sido considerada en el análisis del modelo dinámico del vehículo para la selección de los componentes eléctricos, tales como motor de corriente continua, controlador electrónico, el sistema de frenado regenerativo y baterías. El rendimiento del vehículo eléctrico se evalúa en cuatro condiciones: (i) prueba de frenado, a una velocidad de $13.8 \mathrm{~m} / \mathrm{s}$ el vehículo eléctrico se detiene en $5.1 \mathrm{~m}$; (ii) prueba de potencia en línea recta y a una pendiente del 14\%, se obtuvo $9 \mathrm{~kW}$ y $11 \mathrm{~kW}$, respectivamente; (iii) prueba de frenado regenerativo se recupera en promedio $0.6 \mathrm{~kW}$; y (iv) test de autonomía en la que se consiguió $30.4 \mathrm{~km}$ a plena carga. Se concluye que hay dos componentes principales que deben ser consideradas antes de hacer la conversión EV para lograr mayor eficiencia, la batería y los motores eléctricos.
\end{abstract}

Palabras clave: controlador electrónico; dinámica del vehículo; freno regenerativo; vehículo eléctrico

\section{Evaluation traction system of electric vehicle a two-seat tubular structure}

\begin{abstract}
This article presents an alternative to reduce pollutant emissions from go-kart $7.5 \mathrm{~kW}$ gasoline vehicles in the city of Baños in Ecuador. The proposal is a tubular prototype two-seat electric vehicle that considers design requirements such as: maximum capacity $420 \mathrm{~kg}$, average speed $50 \mathrm{~km} / \mathrm{h}$, climbing slopes of $14 \%$, to maintain occupant comfort. The topography of the city has been considered in the analysis of the dynamic vehicle model for the selection of electrical components such as direct current motor, electronic controller, regenerative braking system and batteries. The performance of electric vehicle is evaluated in four conditions: (i) braking test in a speed of $13.8 \mathrm{~m} / \mathrm{s}$ the electric vehicle stops at $5.1 \mathrm{~m}$; (ii) power test in straight line and one slope of $14 \%$, was obtained $9 \mathrm{~kW}$ and $11 \mathrm{~kW}$ respectively; (iii) regenerative braking test it recovers in average $0.6 \mathrm{~kW}$; and (iv) autonomy test in which $30.4 \mathrm{~km}$ was obtained at maximum load. It is concluded that there are two main components to be considered before making the EV conversion, the battery and electric motors, to achieve maximum efficiency.
\end{abstract}

Keywords: electronic controller; vehicle dynamics; regenerative braking; electric vehicle 


\section{INTRODUCCIÓN}

En los últimos años, los esfuerzos en mejoras tecnológicas e innovación en el transporte se han intensificado y el uso de fuentes renovables en flotas de vehículos son la nueva tendencia para las autoridades públicas, organizaciones no lucrativas y muchas empresas privadas que promueven el desarrollo sostenible (Margaritis et al., 2016). Tomando en cuenta que se recupera energía cinética en el frenado y energía potencial en descenso (Durán et al., 2014). Los vehículos eléctricos son considerados la alternativa más prometedora para los vehículos de motor de combustión interna hacia un sector del transporte más limpio (Casals et al., 2016). De acuerdo a los análisis de la Organización Mundial de la Salud (OMS) 2012-2013, las ciudades del Ecuador sobrepasan los niveles internacionales de contaminación de $10 \mathrm{ug} / \mathrm{m}^{3}$ perjudiciales para la salud, Quito, Milagro y Santo Domingo tienen $18 \mathrm{ug} / \mathrm{m}^{3}, 32 \mathrm{ug} / \mathrm{m}^{3}$ y $33 \mathrm{ug} / \mathrm{m}^{3}$ de $\mathrm{PM}_{2,5}$ respectivamente (OMS, 2016). El desarrollo es continuo en vehículos eléctricos (VE) como: la cadena de tracción, la batería y el cargador han mejorado las tecnologías del VE para la utilización a gran escala (Yong et al., 2015). Los componentes principales del VE son: motor eléctrico, controlador electrónico que consta de inversor, convierten la corriente continua en corriente alterna, el ajuste de la velocidad y el freno (Andrada et al., 2005; Purwadi et al., 2013; Yong et al., 2015).

La actual batería del VE tiene una densidad de energía relativamente baja, lo que afecta directamente a todo el segmento. Además, el coste elevado de la batería ha puesto al VE en una posición de desventaja ya que el coste de compra es considerablemente mayor que un vehículo de motor de combustión interna. También hay preocupaciones sobre el ciclo de vida de la batería y sus características de seguridad (Fotouhi et al., 2016; Kaleg et al., 2015; Yong et al., 2015). El motor eléctrico convencional que se utiliza en el VE comúnmente es de escobillas de corriente continua (DC). En este tipo de motor es fácil el diseño del regulador de velocidad, pero tiene la desventaja de poseer un conmutador mecánico, que provoca la necesidad de mantenimiento. Mientras que un motor de inducción es libre de mantenimiento por no tener un conmutador mecánico, pero el diseño del regulador de velocidad es más complicado (Kaleg et al., 2015).

Una de las técnicas de control que mejores resultados presta al motor eléctrico de corriente alterna, es el control directo de par (CDP), el cual fue introducido hace más de 25 años por Takahashi (1986) en Japón y por Depenbrock (1985) en Alemania. Esta estrategia de control se utiliza ampliamente en los VE (Hegazy et al., 2013). En el control, la aplicación del controlador debe cumplir las siguientes características: i) frecuencia de conmutación fija, utilizando modulación de ancho de pulso (PWM) senoidal; ii) reducción de la distorsión en las corrientes del estator; y iii) menor variación del par generado por el motor. Con esto, el controlador tiene un mejor desempeño que el CDP convencional utilizado comercialmente (Durán et al., 2014). En nuestro trabajo al tener un motor de corriente continua, el control de potencia se logra con las siguientes ventajas i) frecuencia de conmutación PWM que controla transistores mosfet para una configuración en $\mathrm{H}$, para lograr eficiencias del 97\%; ii) inversión de giro; iii) requiere sensor de aceleración, sensor posición (avance y retroceso), sensor activación del freno, posición de llave de encendido; vi) regeneración de energía cuando el motor no reciba carga y al de frenar para las baterías y mayor autonomía.

\section{EL MODELO}

En el VE, el motor de corriente continua o alterna deja de consumir electricidad y comienza a generar, al poseer la alternativa de regeneración al freno, con la ayuda de interfaces para en flujo bidireccional y convertidores que aporta en el sentido de trabajo de las corrientes, como se muestra en la figura 1 (Asamer et al., 2016; Crolla, 2009; Yong et al., 2015).

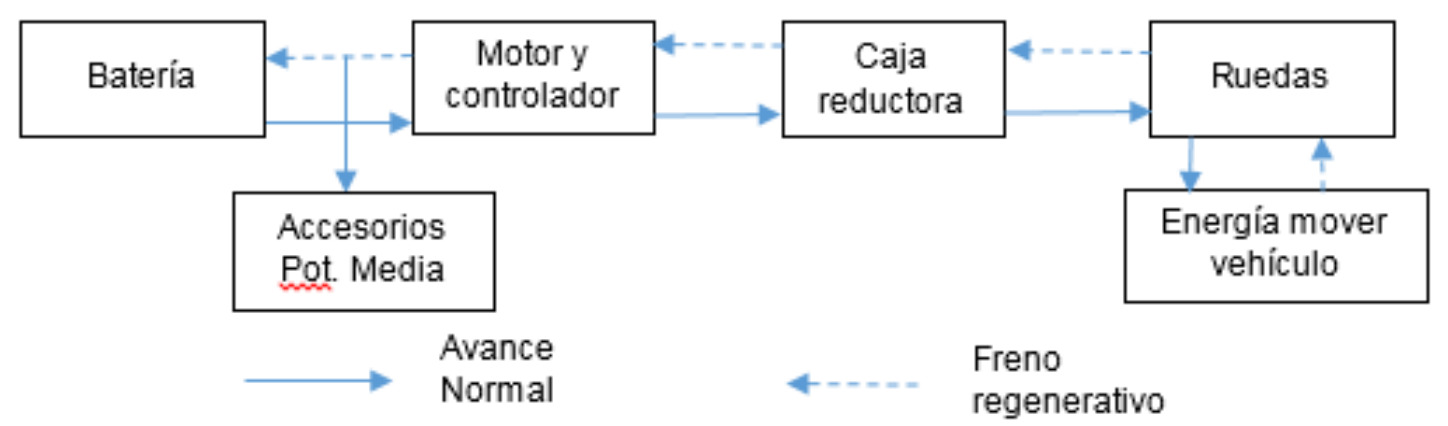

Fig. 1: Diagrama del sistema de recuperación de energía. 


\section{Fuerza de tracción}

Una forma de entender la dinámica de un sistema es mediante un modelo matemático abstracto, el cual es a través de la aplicación de leyes físicas que rigen su comportamiento, el vehículo en pendiente tendrá que cumplir con la Segunda Ley de Newton que plantea que la sumatoria de fuerzas en el eje x, es igual a la masa del cuerpo en movimiento por la aceleración que el vehículo eléctrico tenga, el cual se muestra en la figura 2 y ecuación 1 (Durán et al., 2009; Kaleg et al., 2015; Zhang, 2016).

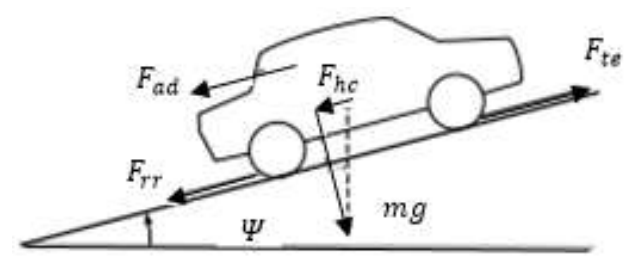

Fig. 2: Fuerzas actuantes sobre el VE en la pendiente.

$F_{t e}=m \cdot a+F_{r r}+F_{a d}+F_{h c}$

En donde: $F_{t e}=$ fuerza de tracción que es la que impulsa al vehículo eléctrico; $m$ = masa; $a$ =aceleración; $F_{r r}=$ fuerza de fricción entre la superficie a rodar y los neumáticos; $F_{a d}=$ fuerza de fricción con el viento; $F_{h c}=$ componente del peso del vehículo a lo largo de la pendiente.

Al relacionar varios parámetros fundamentales, de la ecuación (1) finalmente se tiene la ecuación (2):

$$
F_{t e}=m \cdot a+\mu_{r r} \cdot m \cdot g \cdot \cos (\psi)+\frac{1}{2} \cdot \rho \cdot A \cdot C_{d} \cdot V_{a}^{2}+m \cdot g \cdot \operatorname{sen}(\psi)
$$

En donde: $m=$ masa total (masa del vehículo y masa de la carga); $a=$ aceleración del vehículo; $\mu_{r r}=$ coeficiente de fricción a la rodadura; $g=$ aceleración gravitacional; $\psi=$ ángulo de la pendiente; $\rho=$ densidad aire local; $A=$ área superficie frontal del vehículo; $C_{d}=$ coeficiente aerodinámico; $V_{a}^{2}=$ velocidad del aire al cuadrado.

\section{Potencia del motor}

El cálculo de potencia necesaria en el VE se considera la velocidad máxima en pendiente, para prolongar la autonomía, como se presenta en las siguientes ecuaciones (3) y (4):

$F_{t e}=m \cdot a+\mu_{r r} \cdot m \cdot g \cdot \cos (\psi)+\frac{1}{2} \cdot \rho \cdot A \cdot C_{d} \cdot V_{a}^{2}+m \cdot g \cdot \operatorname{sen}(\psi)$

$P_{\text {calculada }}=F_{\text {te }} \cdot V_{\text {max }}$

Se considera, además un $90 \%$ de eficiencia del motor de corriente continua $\left(\eta_{m}\right)$, del tipo de imanes permanentes con escobillas, y garantizar su trabajo en las distintas condiciones de operación, mediante la expresión (5).

$P_{\text {motor }}=\frac{P_{\text {calculada }}}{\eta_{m}}$

\section{Transmisión}

La relación de transmisión más adecuada en obtener el máximo torque del motor eléctrico (Alcalá et al., 2013). La velocidad angular $(\omega)$ del motor eléctrico puede ser relacionada con la velocidad lineal $(v)$ del vehículo eléctrico y el radio del neumático ( $r$ ) (Alcalá et al., 2013; Durán et al., 2009). Esta relación está dado por (6).

$\omega=\frac{v}{r}$

El torque eléctrico $\left(\tau_{t}\right)$ desarrollado por el motor eléctrico, produce la fuerza de tracción $\left(F_{t}\right)$ del VE, a través de la relación de transmisión $(G)$ establecida, en conjunto al radio del neumático $(r)$, la velocidad angular del neumático $\left(\omega_{n}\right)$, obteniendo la velocidad lineal $(v)$ del vehículo (Alcalá et al., 2013; Rocha et al., 2015), como se presenta en la figura 3 , y expresada en (7).

$\tau_{t}=\frac{F_{t} * r}{G}$ 


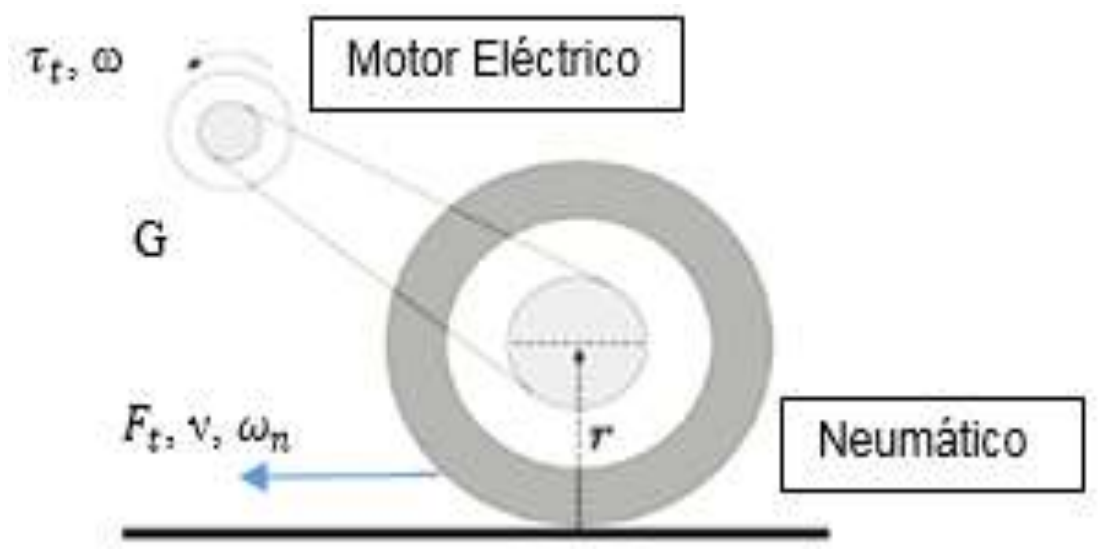

Fig. 3: Torque en la transmisión del VE. (Durán et al., 2009)

\section{Controlador electrónico}

El controlador utiliza un microprocesador que trabaja bajo la estrategia PWM y actúa sobre el conjunto de transistores mosfet de alta potencia dispuesto en forma de puente $\mathrm{H}$, para lograr eficiencias de hasta el $97 \%$ de conmutación en los transistores. El puente $\mathrm{H}$ trabaja en cuatro cuadrantes, en los cuadrantes primero y cuarto, se entrega energía de la batería desarrollando aceleración y recorrido en pendiente, mientras que en el segundo y tercer cuadrante, se realiza la regeneración de la energía mediante la desaceleración y trabajo en vacío, que va a garantizar la recarga de las baterías y correspondiente aumento de autonomía (Durán et al., 2014).

El controlador DC permite un trabajo preciso del motor eléctrico en base a las señales de entrada como: posición del acelerador, sensor de frenado, señal de la batería y selección de giro del motor (avance y retroceso) (Kelly, 2014), como se describe en la figura 4. Las baterías entregan $48 \mathrm{~V}$ para el funcionamiento del motor, el mismo que trabaja en rango de 24/48 V DC.

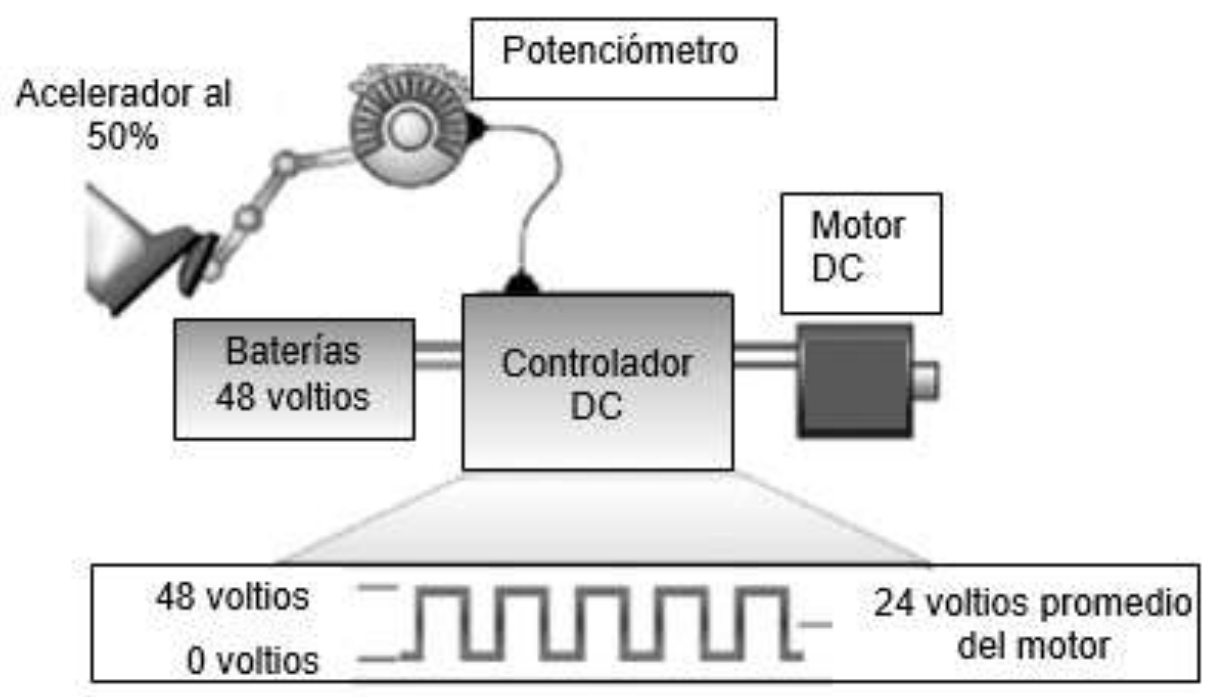

Fig. 4: Gestión electrónica del VE.

\section{RESULTADOS Y DISCUSIÓN}

La estructura que se escoge en la implementación para la propulsión eléctrica es uno de tipo tubular, modelo YH150A. Dando resultado de forma experimental los parámetros de la tabla 1. Según George A. D., \& Besselink I. (2015), en el diseño también se mitiga algunos problemas de la alta masa no suspendida. El prototipo se visualiza en la figura 5. Se evaluó el desempeño del vehículo eléctrico en cinco condiciones: prueba de frenado, prueba de potencia entregada en línea recta y en pendiente al 14\%, prueba de frenado regenerativo y prueba de autonomía. 


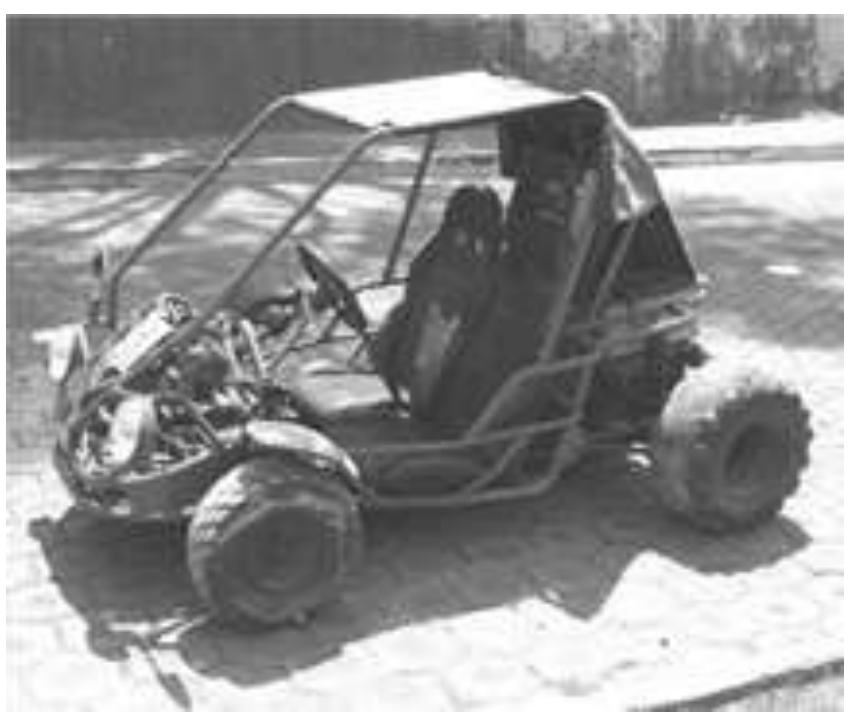

Fig. 5: Prototipo vehículo eléctrico.

Tabla 1: Parámetros aplicados en el vehículo eléctrico para las pruebas.

\begin{tabular}{|c|c|}
\hline \multicolumn{2}{|c|}{ Características del vehículo eléctrico } \\
\hline m: Masa Vehículo Eléctrico & $420 \mathrm{~kg}$ \\
\hline$C_{\boldsymbol{d}}:$ Coeficiente aerodinámico & 0.6 \\
\hline$R$ : Radio del neumático & $0.25 \mathrm{~m}$ \\
\hline$f_{r}:$ Coeficiente de resistencia a la rodadura & 0.015 \\
\hline$G:$ Relación de transmisión & 4.08 \\
\hline$A$ : Área de la superficie frontal del vehículo & $2.03 \mathrm{~m}^{2}$ \\
\hline \multicolumn{2}{|c|}{ Características del motor eléctrico } \\
\hline$P_{m}:$ Potencia del motor & 12 Hp (9 kW) @ 3200 rpm \\
\hline$\tau_{e}:$ Torque del motor & 27.15 Nm @ 48 V DC \\
\hline$\omega$ Velocidad angular & 2600 rpm \\
\hline$V_{I}:$ Voltaje de trabajo & 48 V DC \\
\hline$I_{I}:$ Corriente máxima & $400 \mathrm{~A} / \mathrm{min}$. \\
\hline$\eta_{m}:$ Eficiencia eléctrica del motor & $90 \%$ \\
\hline \multicolumn{2}{|c|}{ Características del controlador } \\
\hline Eficiencia controlador & $97 \%$ \\
\hline IGBT: Módulo Kelly PM72401B & $48 \mathrm{~V} \mathrm{DC} / 120 \mathrm{~A}$ y máximo $400 \mathrm{~A}$. \\
\hline \multicolumn{2}{|c|}{ Características de la Batería } \\
\hline Tipo & Ciclo profundo \\
\hline Voltaje & 12 V DC \\
\hline Peso & $37 \mathrm{~kg}$ \\
\hline Capacidad & $120 \mathrm{Ah}$ \\
\hline Capacidad específica & $38.92 \mathrm{Wh} / \mathrm{kg}$ \\
\hline
\end{tabular}

La prueba de frenado se considera distintas velocidades, en un tiempo y distancia evaluadas hasta completar la prueba, en la figura 6 , se presenta los datos recomendados en la conducción del VE. Los valores de velocidad máxima y distancia de frenado obtenidos son $13.8 \mathrm{~m} / \mathrm{s}$ y $5.1 \mathrm{~m}$ respectivamente, se evidencia que es proporcional la distancia de frenado y a la velocidad experimentada. 


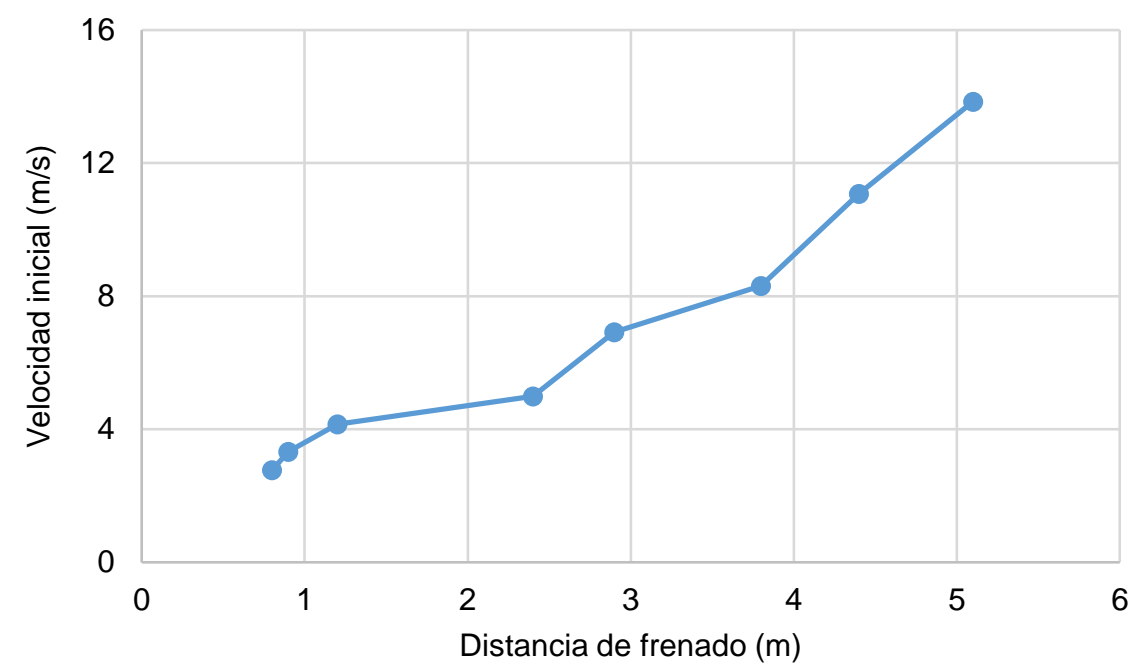

Fig. 6: Distancia de frenado vs Velocidad del VE.

Según Kaleg et al. (2015), para obtener mayor autonomía usando el mismo sistema de tensión, la capacidad de la batería debe ser ampliada. Esto evita que el motor eléctrico entregue el mismo rendimiento; Sin embargo su autonomía aumentará. Una velocidad más alta se puede obtener mediante el uso de un motor eléctrico de alto rendimiento, produciendo aumento de tensión. De los datos analizados en las pruebas de recorrido, se tiene que la caída de voltaje máxima se obtiene al acelerar en línea recta es de $44 \mathrm{~V}$., y de la prueba en pendiente ascendente al $14 \%$, es la más exigente de la prueba realizada en la ciudad de Baños es de 43.6 V., al hacerlo al $100 \%$ de aceleración.

En la figura 7, se presenta la potencia desarrollada por el motor eléctrico a dos condiciones de prueba, en línea recta y en pendiente ascendente del 14\%. La línea de puntos representa el trabajo desarrollado en línea recta por el VE, se obtiene un consumo de $2.2 \mathrm{~kW}$ y $9.0 \mathrm{~kW}$ al $25 \%$ y $100 \%$ del acelerador respectivamente. La línea de cruces, representa el trabajo desarrollado en pendiente ascendente al $14 \%$, se obtiene $2.3 \mathrm{~kW}$ a $11 \mathrm{~kW}$ al $25 \%$ y $100 \%$ del acelerador respectivamente. Dando lugar, el consumo es directamente proporcional a la resistencia mecánica del trayecto del VE.

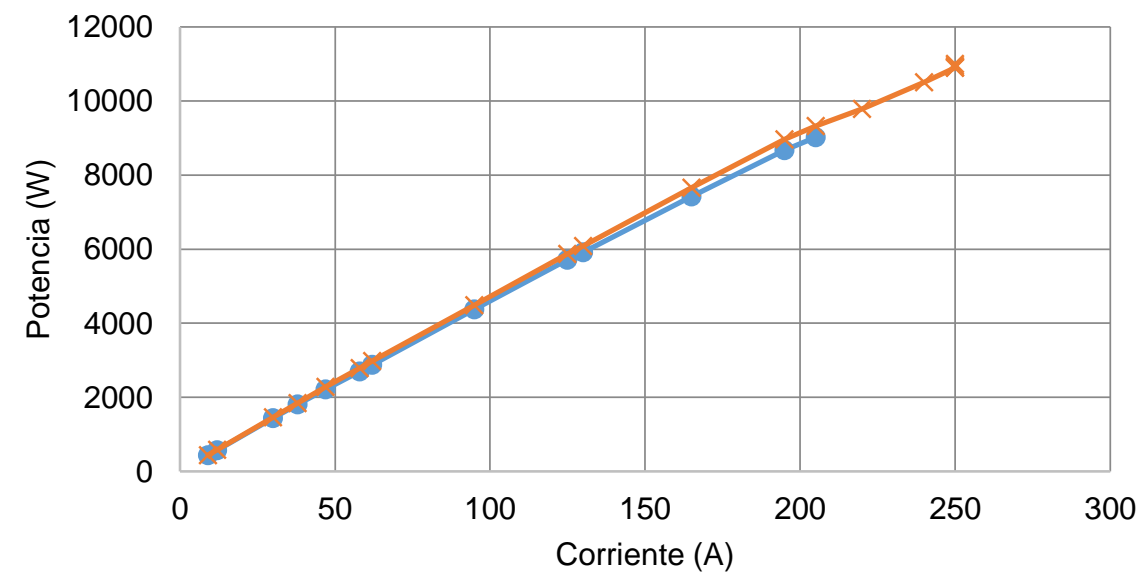

Fig. 7: Potencia del motor eléctrico entregada al VE: línea de puntos, desarrollada en línea recta; línea de cruces ${ }^{*}$ desarrollada en pendiente al $14 \%$.

Según Alcalá et al. (2014), durante el período de desaceleración el sistema regenerativo puede recuperar una cierta cantidad de energía que se almacena para ser utilizada en el periodo de aceleración. Esto es corroborado por los resultados en este trabajo. En la figura 8 se muestra la regeneración que se obtiene en el sistema cuando el VE se desplaza en una trayectoria con pendiente negativa del $14 \%$ (descenso), entregando el sistema una corriente máxima de $12.1 \mathrm{~A}$, a $49.5 \mathrm{~V}$, lo que da una potencia de $0.6 \mathrm{~kW}$ disponible para ser usada por el VE. Esto quiere decir que el motor en lugar de consumir corriente la está entregando a las baterías convirtiéndose en generador. 


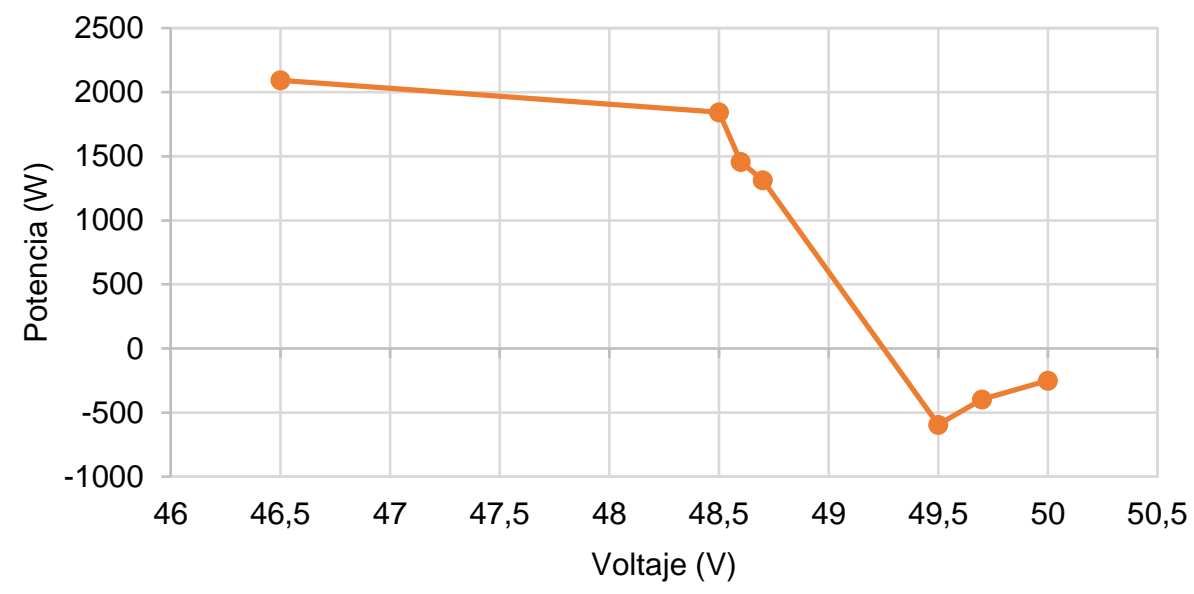

Fig. 8: Prueba del Freno Regenerativo, potencia vs voltaje.

La ruta utilizada para determinar la autonomía del VE, tiene las siguientes características: distancia total 15.6 $\mathrm{km}$, distancia descenso $7.6 \mathrm{~km}$ (pendiente 14\%), distancia en ascenso $8 \mathrm{~km}$, (pendiente de 14\%). En esta ruta con el banco de baterías cargado completamente, el VE en promedio recorrió una distancia de $30.4 \mathrm{~km}$ a plena carga, y $10 \mathrm{~km}$ adicionales a carga mínima.

\section{CONCLUSIONES}

El análisis dinámico del vehículo permitió la selección de los principales componentes del vehículo eléctrico biplaza de estructura tubular, tales como el motor de corriente continua de imanes permanentes con escobillas de $9 \mathrm{~kW}$, controlador electrónico de $48 \mathrm{~V} \mathrm{DC} / 120 \mathrm{~A}$ y su adaptación de trabajo a un sistema de freno regenerativo, además de un sistema de baterías de $48 \mathrm{~V}$, permitiendo al prototipo alcanzar una velocidad media de $50 \mathrm{~km} / \mathrm{h}$, desplazar una carga máxima de $420 \mathrm{~kg}$ y vencer pendientes de $14 \%$ evaluadas en el recorrido de prueba.

Se realizaron 5 evaluaciones al prototipo considerando la topografía de la ciudad obteniendo los siguientes resultados: Prueba de frenado, a una velocidad de $13.8 \mathrm{~m} / \mathrm{s}$ el vehículo eléctrico se detiene en $5.1 \mathrm{~m}$; Prueba potencia, en línea recta y en pendiente ascendente del 14\%, el vehículo experimentó un valor máximo de 9.0 kW y $11 \mathrm{~kW}$ respectivamente al $100 \%$ de aceleración desarrollada por el motor eléctrico; La prueba de regeneración a una pendiente negativa del $14 \%$ se evidenció un aporte instantáneo en el sistema de baterías de $0.6 \mathrm{~kW}$ en promedio; La prueba de autonomía, el prototipo realizó un recorrido $7.6 \mathrm{~km}$ en descenso y 8 $\mathrm{km}$ en ascenso consiguiendo un total de dos recorridos a plena carga más $10 \mathrm{~km}$ adicionales a carga mínima.

Al considerar una autonomía de $40 \mathrm{~km}$ por litro de combustible del go-kart de $7.5 \mathrm{~kW}$. La alternativa de conversión de los vehículos de gasolina de la ciudad de Baños a vehículos eléctricos permite reducir las emisiones de dióxido de carbono en un valor de $2.2 \mathrm{~kg}$ de $\mathrm{CO} 2$ por cada litro de combustible consumido.

\section{REFERENCIAS}

Alcalá, I. y otros cuatro autores, Electric Vehicle Emulation Based on Inertial Flywheel and a Dc Machine, doi:dx.doi.org/10.15446/dyna.v81n183.31638, Dyna rev. fac. nac. minas, 81 (183), 86 - 96 (2014)

Andrada, P., M. Torrent, B. Blanqué, y J. Perat, Accionamientos de Reluctancia Autoconmutados para Vehículos Eléctricos, doi:dx.doi.org/10.4067/S0718-07642005000500013, Inf. Tecnol., 16(5), 79-87 (2005)

Asamer, J. y otros tres autores, Sensitivity Analysis for Energy Demand Estimation of Electric Vehicles, doi:10.1016/j.trd.2016.03.017, Transportation Research Part D, 46, 182 - 199 (2016)

Casals, L.C., E. Martinez-Laserna, B. A. García y N. Nieto, Sustainability Analysis of the Electric Vehicle Use in Europe for $\mathrm{CO}_{2}$ Emissions Reduction, doi:10.1016/j.jclepro.2016.03.120, Journal of Cleaner Production, $127,425-437$ (2016)

Crolla D., Automotive Engineering, $1^{\text {a }}$ edición, p.p. 155-156, Elsevier Inc., Burlington, USA (2009)

Depenbrock, M., Direkte Selbstregelung (DSR) fiir hochdynamische Drehfeld-antriebe mit Stromrichterschaltung, ETZ A 7, 211-218 (1985) 
Durán M., G. Guerrero-Ramírez y A. Claudio, Determinación de los Requerimientos de Par, Velocidad Angular y Potencia para el Motor de un Vehículo Eléctrico, Congreso Anual 2009 de la Asociación de México de Control Automático, 401 - 407, Zacatecas, México, 30 septiembre a 02 octubre (2009)

Durán, M.A., J. Gudiño, S. M. Charre y J. A. Alcalá, Modelado y Diseño del Controlador para un Sistema de Tracción de un Vehículo Eléctrico, doi:dx.doi.org/10.4067/S0718-07642014000600013, Inf. Tecnol., 25 (6), 101-108 (2014)

Fotouhi A., D. J. Auger, K. Propp, S. Longo y M. Wild, A Review on Electric Vehicle Battery Modelling: From Lithium-Ion Toward Lithium-Sulphur, Renewable and Sustainable Energy Reviews, 56, 1008 - 1021 (2016)

George A. D., e I. Besselink, Rear Suspension Design for An In-Wheel-Drive Electric Car, doi:10.1177/ 0954407015581938, Proceedings of the Institution of Mechanical Engineers, Part D, Journal of Automobile Engineering, 1-13 (2015)

Hegazy O., y otros cinco autores, Control, Analysis and Comparison of Different Control Strategies of Electric Motor for Battery Electric Vehicles Applications, doi:10.1109/EPE.2013.6631906, Power Electronics and Applications (EPE), 15 th European Conference, 1-13 (2013)

Kaleg S., A. Hapid y M.R. Kurnia, Electric Vehicle Conversion Based on Distance, Speed and Cost Requirements, doi:10.1016/j.egypro.2015.03.276, Energy Procedia, 68, 446 - 454 (2015)

Kelly Controls LCC, User's Manual (en línea), https://kellycontroller.com/mot/downloads/KDZGDZUserManual.pdf. Acceso: 20 de mayo (2016), Kelly Controls LCC, USA (2014)

Margaritis D., A. Anagnostopoulou, A. Tromaras y M. Boile, Electric Commercial Vehicles: Practical Perspectives and Future Research Directions, doi:10.1016/j.rtbm.2016.01.005, Research in Transportation Business \& Management, 18, 4 - 10 (2016)

OMS, Fichas de las ciudades más contaminadas del Ecuador (en línea), http://www.elcomercio.com/tendencias/ciudades-ecuador-polucion-enfermedades-contaminacion.html.

Acceso: 10 de septiembre (2016), El comercio, EC (2016)

Purwadi A., J. Dozeno y N. Heryana, Testing Performance of $10 \mathrm{~kW}$ BLDC Motor and LiFePO 4 Battery on ITB-1 Electric Car Prototype, doi:10.1016/j.protcy.2013.12.296, Procedia Technology, 11, 1074 - 1082 (2013)

Rocha H. J. y otros cinco autores, Diseño e Implementación del Sistema de Tracción y Control Eléctrico para un Cross Car Biplaza Turístico, Memorias Congreso Nacional de Ingeniería Automotriz y Desagregación Tecnológica 2015, ISBN: 978-9978-10-220-6 (CD), 1(1), 251-260 (2015)

Takahashi I., y T. Noguchi, A New Quick-Response and High Efficiency Control Strategy of an Induction Motor, doi:110.1109/TIA.1986.4504799, IEEE Trans. Ind. Appl., IA-22 (5), 820 - 827 (1986)

Yong J. Y., V.K., Ramachandaramurthy, K.M., Tan y N.A. Mithulananthan, A Review On the State-of-the-Art Technologies of Electric Vehicle, Its Impacts and Prospects, doi:10.1016/j.rser.2015.04.130, Renewable and Sustainable Energy Reviews, 49, 365 - 385 (2015)

Zhang R. y E. Yao, Electric Vehicles' Energy Consumption Estimation with Real Driving Condition Data, Transportation Research Part D: Transport and Environment, 41, 177 - 187 (2015) 